\title{
Desempenho inicial de plântulas de soja oriundas de sementes quimicamente tratadas
}

\section{Initial performance of soybean seedlings derived from chemically treated seed}

\author{
Rafael de Oliveira Vergara ${ }^{*}$, Henrique Roberto Maldaner ${ }^{2}$, Vanessa Nogueira Soares ${ }^{3}$, Gizele Ingrid Gadoti ${ }^{4}$, Lilian \\ Madruga Tunes ${ }^{5}$
}

Resumo: A soja atualmente é um dos principais produtos do agronegócio brasileiro. Diversos fatores afetam a produtividade da cultura, entre eles o número de plantas estabelecidas merece devido destaque. Grande parte dos produtores brasileiros utiliza o tratamento químico de sementes com objetivo de melhorar o estande inicial de plantas. Neste trabalho se objetivou avaliar o desempenho inicial de sementes de soja tratadas quimicamente com os principais produtos disponíveis no mercado. Utilizou-se dois lotes da cultivar BMX Turbo RR, com distintos níveis de vigor, estes foram submetidos aos três principais tratamentos químicos do mercado. Foram avaliada emergência, índice de velocidade de emergência, comprimento e biomassa seca de raiz, área foliar. Ocorreu diferença entre os tratamentos, no lote de baixo vigor o tratamento quatro (fludioxonil, metalaxylm e tiametoxan) melhorou o desempenho, em sementes de alto vigor o tratamento não favoreceu o desempenho das sementes.

Palavras-chave: Glycine max; Estande inicial; Tratamento químico

\begin{abstract}
Soy is currently one of the main products of the Brazilian agribusiness. Several factors affect the productivity of the culture, including the number of established plants deserves due attention. Most Brazilian producers use chemical seed treatment in order to improve the plant stand. This work aimed to evaluate the initial performance of soybean seeds chemically treated with leading products on the market. We used two batches of growing BMX Turbo RR, with different levels of vigor, these were subjected to three main chemical treatments market. emergency were evaluated, emergency speed index, length and root dry biomass, leaf area. Was no difference between treatments in the lot of low force treatment four (fludioxonil, metalaxylm and Thiamethoxam) improved performance in high vigor seed treatment did not improve the performance of seeds.
\end{abstract}

Key words: Glycine max; Initial stand; Chemical treatment

\footnotetext{
*Autor para correspondência

Recebido para publicação em 18/05/2016; aprovado em 20/11/2016

${ }^{1}$ Engenheiro Agrônomo, Mestrando em Ciência e Tecnologia de Sementes, UFPel, Pelotas; Fone: 5399921456, agrorafaelvergara@ gmail.com

${ }^{2}$ Graduando em agronomia, UFPel, henrique-maldaner@hotmail.com

${ }^{3}$ Doutora em Ciência e Tecnologia de Sementes, UFPel, vnsoares@ gmail.com

${ }^{4}$ Doutora em Ciência e Tecnologia de Sementes, UFPel, gizeleingrid@gmail.com

${ }^{5}$ Doutora em Ciência e Tecnologia de Sementes, UFPel, lilianmtunes@yahoo.com
} 


\section{INTRODUÇÃO}

A soja (Glycine max) é a principal oleaginosa cultivada no mundo, o alto uso desta na produção de biocombustíveis, alimento e indústria do petróleo exige o aumento da sua produtividade (SEGALIN et al., 2013). É uma das principais commodities do agronegócio brasileiro, semeada em grande parte dos campos de produção do país. Desta forma toda pratica que tenha por objetivo o aumento de produção e rentabilidade da referida cultura merece o devido destaque.

A qualidade das sementes é fator primordial para estabelecimento de lavouras com alto potencial produtivo. Sementes de alto vigor produzem plantas com maior velocidade de emergência e maiores níveis nos componentes de rendimento, desdobrando assim em uma maior produtividade de sementes no final do seu ciclo (SILVA et al., 2016). São diversos os fatores que limitam a produtividade na cultura da soja, entre eles podemos citar a ocorrência de doenças e pragas durante o estabelecimento da cultura, causando a diminuição na população de plantas.

Condições climáticas adversas podem comprometer o desenvolvimento inicial da cultura, em situações desfavoráveis à germinação e emergência o tratamento de sementes de soja é uma prática essencial para assegurar estande adequado, além de reduzir a possibilidade de introdução de patógenos em áreas indenes (PEREIRA et al., 2011).

Tendo em vista a possível ocorrência de pragas e doenças que podem afetar negativamente o estabelecimento da cultura, medidas de controle devem ser adotadas. Dentre essas medidas, o uso de cultivares resistentes, sementes livres de patógenos e o tratamento químico podem garantir a obtenção de plantas mais sadias e produtivas (MERTZ et al., 2009). Com o objetivo de proteger as sementes e as plântulas na fase de estabelecimento contra todo tipo de adversidades, produtos fitossanitários como fungicidas e inseticidas são aplicados às sementes (LUDWIG et al., 2011).

Segundo Avelar et al. (2011) o tratamento de sementes de soja é uma técnica economicamente recomendada, desde que utilizados produtos ou misturas de produtos adequados, na dosagem correta e distribuídos uniformemente em todo o lote de sementes. A utilização de tratamento químico de sementes é uma técnica atualmente disseminada entre os produtores de soja brasileiros, visando à proteção da semente durante o desenvolvimento inicial.

O tratamento de sementes de soja com os produtos químicos mantém a qualidade fisiológica, genética e sanitária destas, apresentando efeitos benéficos em diversas fases do crescimento inicial e do desenvolvimento da cultura, porém sem efeito sobre a produção de grãos em plantas de soja (CUNHA et al., 2015). Entretanto Ludwig et al. (2011) enfatiza a redução da germinação com a aplicação de fungicida e/ou inseticida, relacionando com a ação do ingrediente ativo sobre as sementes, que pode acarretar um efeito fitotóxico e redução da germinação.

Assim o objetivo do presente trabalho foi de avaliar o desempenho inicial de sementes de soja submetidos aos principais tratamentos químicos atualmente utilizados.

\section{MATERIAL E MÉTODOS}

O trabalho foi conduzido durante o mês de outubro de 2015, no Laboratório Didático de Análise de Sementes do
Programa de Pós Graduação em Ciência e Tecnologia de Sementes.

Foram utilizados dois lotes de sementes de soja da cultivar BMX TURBO RR, pertencente ao grupo de maturação 5.9 e atualmente é uma das mais semeadas na região sul do Rio Grande do Sul. Estes apresentando dois níveis de vigor através do teste de envelhecimento acelerado.

Nas sementes foram aplicados os principais tratamentos comerciais utilizados atualmente (Tabela 1), constituindo três tratamentos e um controle (sementes não tratadas). As doses utilizadas foram as recomendadas pelos fabricantes, para a aplicação dos produtos foi ajustado o volume de calda, de forma que todos os tratamentos apresentassem o mesmo volume aplicado.

Tabela 1. Tratamentos comerciais aplicados a dois lotes de sementes de soja da cultivar BMX TURBO RR.

\begin{tabular}{ccc} 
Tratamento & Ingrediente ativo & $\begin{array}{c}\text { Dose } \\
\left(\mathrm{mL}^{*} 100 \mathrm{~kg}^{1}\right)\end{array}$ \\
\hline 1 & $\begin{array}{c}\text { Piraclostrobina+tiofanato } \\
\text { metílico+ fipronil }\end{array}$ & - \\
2 & $\begin{array}{c}\text { Imidacloprido+tiodicarbe+c } \\
\text { arben } \\
\text { dazim+tiram }\end{array}$ & 700 \\
4 & $\begin{array}{c}\text { Fludioxonil+metalaxylm+ } \\
\text { Tiametoxan }\end{array}$ & 300 \\
\hline
\end{tabular}

$\mathrm{O}$ efeito dos tratamentos foram mensurados pelas variáveis descritas a seguir:

Emergência a campo: a semeadura foi feita em solo oriundo de campo de produção da cultura, utilizando-se cinco repetições de cinquenta sementes para cada tratamento. A contagem das plântulas emergidas foi realizada aos quatorze dias após a semeadura. $\mathrm{O}$ resultado foi expresso em porcentagem de plântulas emergidas.

Índice de velocidade de emergência: foi conduzido em conjunto com a emergência a campo, foram efetuadas contagens diárias observando-se o número de plântulas emergidas. O índice foi calculado empregando-se a fórmula proposta por Maguire (1962).

Comprimento de raiz: aos 21 dias após semeadura foram cuidadosamente coletadas dez plântulas por repetição de cada tratamento. Com a utilização de régua milimetrada foi mensurado a distância desde o nível do solo até a maior distância de raiz. Foi calculada a média do comprimento de raiz das plântulas de cada tratamento e o resultado foi expresso em centímetros de raiz.

Biomassa seca de raiz: foi determinada após a determinação do comprimento de raiz. Assim, as raízes das plântulas das repetições de cada lote foram acondicionadas em sacos de papel e levadas a estufa termoelétrica, mantida à temperatura de $65^{\circ} \mathrm{C}$, permanecendo por 72 horas, quando atingiu peso constate. Após esse período, o material foi resfriado em dessecador e as raízes das plântulas de cada repetição foram pesadas individualmente em balança de precisão de 0,0001g (NAKAGAWA, 1999). Foi calculada a média da biomassa seca de raiz das plântulas de cada tratamento e o resultado foi expresso em gramas.

Área foliar: aos 21 dias após a semeadura, foram coletadas dez plantas oriundas de cada tratamento e desta 
foram avaliadas área foliar em aparelho LI-3000. Resultado expresso em $\mathrm{dm}^{2}$.

Dano mecânico (hipoclorito de sódio): realizada de acordo com Krzyzanowski et al. (2004), foram utilizadas quatro subrepetições de 50 sementes, estas foram imersas durante 10 minutos numa solução diluída de hipoclorito de sódio, com concentração de $0,2 \%$. Posteriormente, o excesso da solução foi eliminado e as sementes distribuídas sobre folhas de papel toalha para determinação da porcentagem de sementes intumescidas (danificadas). Os resultados foram expressos em porcentagem.

$\mathrm{O}$ delineamento experimental utilizado foi inteiramente casualizado. Os dados foram submetidos à ANOVA, a comparação de médias pelo teste Tukey e análise de correlação

\section{RESULTADOS E DISCUSSÃO}

O índice de velocidade de emergência (Tabela 2) foi influenciado pelo tratamento químico utilizado nas sementes, o efeito foi observado nos dois lotes estudados. Para o lote 1, considerado de baixo vigor, o tratamento 2 e 3 não afetaram a variável quando comparado a semente não tratada, entretanto o tratamento 4 melhorou o desempenho do lote para a variável em questão. No lote 2 , considerado de alto vigor, a utilização de tratamento químico afetou de forma negativa a variável em questão, vale ressaltar que o tratamento 4 apresentou o pior desempenho. Entretanto Dan et al.(2010) estudando diferentes tratamentos, observou que não existe diferença significativa entre a utilização dos princípios ativo fipronil e thiamethoxan, os referidos também não diferem da semente não tratada, assim estes não afetaram a velocidade de emergência.

A variável emergência a campo foi influenciada pelo tratamento nos diferentes lotes. No lote 1, o tratamento 4 apresentou desempenho superior aos demais, entretanto não diferiu estatisticamente dos tratamentos 1 e 2, o tratamento 3 apresentou pior desempenho que os demais, entretanto estatisticamente não diferiu dos tratamentos 1 e 2 . O melhor desempenho do tratamento 4 pode ser atribuído ao ingrediente ativo tiametoxam presente na sua formulação, Almeida et al. (2011) estudando o tratamento de sementes de arroz com o referido principio ativo, concluiu que este favorece positivamente a qualidade fisiológica das sementes tratadas. Para o lote 2, o controle apresentou desempenho superior aos tratamentos 3 e 4, não diferindo estatisticamente apenas do tratamento 2, entretanto numericamente o tratamento químico influenciou de forma negativa a variável, resultado semelhante foi encontrado por Castro et al.(2008), este observou que a utilização de tratamento químico em ambiente controlado aumenta o percentual de plântulas anormais e mortas.

Tabela 2. Dados referentes a índice de velocidade de emergência (IVE), emergência (E), comprimento de raiz (CR), área foliar (AF), biomassa seca de raiz (BS)

\begin{tabular}{|c|c|c|c|c|c|}
\hline & \multicolumn{5}{|c|}{ LOTE 1} \\
\hline & IVE & $\mathrm{E}(\%)$ & BS (g) & $\mathrm{CR}(\mathrm{cm})$ & $\mathrm{AF}\left(\mathrm{dm}^{2}\right)$ \\
\hline T 1 & $7,96 \mathrm{ab}^{*}$ & $86 \mathrm{ab}^{*} \mathrm{~B} * *$ & $0,82 \mathrm{a}^{*}$ & $18,26 \mathrm{a}^{*}$ & $2.78^{\mathrm{ns}}$ \\
\hline Т 2 & $7,52 \mathrm{ab}^{*}$ & $84 a b^{*}$ & $0,4 \mathrm{~b}^{*}$ & $17,25 \mathrm{ab}^{*}$ & 2,87 \\
\hline T 3 & $7,24 b^{*}$ & $82 b^{*}$ & $0,66 \mathrm{ab}^{*}$ & $13,10 b^{*}$ & 2,49 \\
\hline $\mathrm{T} 4$ & $8,24 \mathrm{a}^{*}$ & $91 \mathrm{a}^{*}$ & $0,6 \mathrm{ab}^{*}$ & $17,04 \mathrm{ab}^{*}$ & 2,93 \\
\hline \multirow[t]{3}{*}{$\mathrm{CV}(\%)$} & 4,94 & 4,06 & 25,91 & 14,25 & 19,75 \\
\hline & \multicolumn{5}{|c|}{ LOTE 2} \\
\hline & IVE & $\mathrm{E}(\%)$ & BS (g) & $\mathrm{CR}(\mathrm{cm})$ & $\mathrm{AF}\left(\mathrm{dm}^{2}\right)$ \\
\hline $\mathrm{T} 1$ & $8,76 \mathrm{a}^{*}$ & $94 \mathrm{a}^{*} \mathrm{~A}^{* *}$ & $0,85 \mathrm{a}^{* *}$ & $17,47 \mathrm{a}^{* *}$ & $2,83^{\mathrm{ns}}$ \\
\hline $\mathrm{T} 2$ & $7,57 \mathrm{bc}^{*}$ & $86 \mathrm{ab}^{*}$ & $0,44 \mathrm{c}^{* *}$ & $17,6 \mathrm{a}^{* *}$ & 2,65 \\
\hline T 3 & $6,74 c^{*}$ & $80 b^{*}$ & $0,51 \mathrm{bc}^{* *}$ & $14,1 \mathrm{~b} * *$ & 2,52 \\
\hline $\mathrm{T} 4$ & $7,71 b^{*}$ & $79 b^{*}$ & $0,67 a b * *$ & $17,2 \mathrm{a} * *$ & 3,1 \\
\hline $\mathrm{CV}(\%)$ & 5,98 & 9,42 & 16,83 & 5,71 & 14,98 \\
\hline
\end{tabular}

Médias seguidas pela mesma letra minúscula na coluna ou maiúscula na linha não diferem estatisticamente por Tukey, com nível de $5 \%(*)$ ou $1 \%(* *)$ de significância.

Para a variável biomassa seca de raiz, ocorreu efeito significativo para os tratamentos. Observando o lote 1 , o tratamento 1 (controle) favoreceu a variável, diferindo estatisticamente do tratamento 2 , os demais tratamentos não diferiram estatisticamente. Para o lote 2, o efeito foi mais evidente, apresentando o controle como tratamento de melhor desempenho e o tratamento 2 como de pior desempenho para a variável.

A variável comprimento de raiz (Tabela 2), foi afetada pelo tratamento químico nos diferentes lotes. Para o lote 1 , a não utilização de tratamento químico favoreceu o desenvolvimento do sistema radicular, entretanto o tratamento 1 (controle), não diferiu dos tratamentos 2 e 4, portanto não afetaram de forma negativa a variável, para o referido lote o tratamento 3 suprimiu o desenvolvimento da raiz. No lote 2 a variável foi favorecida pelos tratamentos 1,2 e 4 . Resultado distinto foi observado por Balardin et al.(2011), avaliando diferentes tratamentos de sementes, indicou que não ocorre diferença entre os produtos utilizados quando se compara a variável comprimento de raiz. Entretanto Vanzolini et al.,(2007), estudando a relação entre o desempenho inicial de plântulas de soja, concluiu que o comprimento da raiz de plântulas apresenta maior sensibilidade para verificar a qualidade fisiológica, apresentando correlação significativa com a emergência a campo.

A variável área foliar não foi afetada pelo tratamento nos diferentes lotes, fato este pode ser atribuído à condução do teste, este foi conduzido em condições favoráveis sem restrição hídrica e ataque de pragas. Assim a eficiência dos diferentes princípios ativo no controle de insetos e doenças não foi colocado a prova, este experimento tem como objetivo mensurar a influencia na qualidade fisiológica da utilização 
do tratamento químico nos lotes estudados. Estudando o desempenho de sementes tratadas com diferentes produtos químicos, Cunha et al.(2015) encontrou resultado semelhante, assim os tratamentos utilizados não influenciaram a área foliar durante o desenvolvimento inicial da cultura.

Observando os dados presentes na tabela 3, é evidente a existência de correlação significativa (5\%), entre as variáveis índice de velocidade de emergência e peso de mil sementes. Neste caso o aumento no peso de mil sementes ocasiona no declínio no índice de velocidade de emergência. Tal fato pode ser relacionado à diminuição da área superficial da massa de sementes ocasionado pelo aumento de tamanho destas, assim ocorrendo maior deposição de ingrediente ativo por unidade de área. Abati et al.(2014) relatam a ocorrência de fitotoxidez em plântulas geradas a partir de sementes tratadas, ocasionando a diminuição da qualidade fisiológica destas. Assim com a diminuição relativa da área da massa de sementes e com o aumento da concentração de ingrediente ativo sobre as sementes, tal efeito pode ser mais evidente, ocasionando diminuição no vigor do lote.

O aumento na ocorrência de dano mecânico ocasionou diminuição nas variáveis emergência e comprimento de raiz (Tabela 3), apresentando respectivamente 5 e $1 \%$ de significância. Tal relação deve ser atribuída a lesão ocasionada pelo dano, ocasionando a maior atividade respiratória e maior exposição dos tecidos a fatores externos, assim culminando no declínio na qualidade do lote. Diversos autores relacionam o declínio na qualidade fisiológica de sementes com o nível de ocorrência de dano mecânico, a intensidade na perda de qualidade esta relacionada com a profundidade e a localização do dano (MONDO et al., 2009; SOUZA et al., 2009).

Vale ressaltar que os dados não são conclusivos, para obtenção de resultados mais específicos devem ser efetuados novos testes. Entretanto tais observações servem de indicio para demonstrar a necessidade de estudos neste sentido.

Tabela 3. Correlação de Pearson para sementes quimicamente tratadas, índice de velocidade de emergência (IVE), emergência (E), matéria seca de plântula (MS), peso de mil sementes(PMS), comprimento de raiz (CR)

\begin{tabular}{lcccc}
\hline & $\mathrm{AF}\left(\mathrm{dm}^{2}\right)$ & $\mathrm{DM}(\%)$ & $\mathrm{PMS}(\mathrm{g})$ & $\mathrm{CR}(\mathrm{cm})$ \\
\hline $\mathrm{IVE}$ & -0.9066 & 0.28840 & $-0.91282^{* *}$ & -0.57384 \\
$\mathrm{E}(\%)$ & -0.10997 & $-0.81818^{*}$ & 0.06802 & $0.82827^{*}$ \\
$\mathrm{MS}(\mathrm{g})$ & $0.9649^{* *}$ & 0.32567 & 0.48326 & 0.079698 \\
$\mathrm{DM}(\%)$ & & & -0.49171 & $-0.90071^{* *}$ \\
\hline
\end{tabular}

* significativo em nível de 5\% de significância

** significativo em nível de $1 \%$ de significância

\section{CONCLUSÕES}

Existe diferença entre os tratamentos testados no desempenho inicial de sementes de soja.

Em sementes de baixo vigor a utilização dos princípios ativos fludioxonil, metalaxylm e tiametoxan, favorecem o desempenho inicial.

Sementes de alto vigor não são favorecidas pela utilização de tratamento químico nas condições testadas.

\section{REFERÊNCIAS}

ABATI, J.; ZUCARELI, C.; FOLONI, J. S. S.; HENNING, F. A.; BRZEZINK, C. R.; HENNING, A. A. Treatment with fungicides and insecticides on the physiological quality and health of wheat seeds. Journal of Seed Science, Londrina, v.36, n.4, p.392-398, 2014.

ALMEIDA, A. S.; CARVALHO, I.; DEUNER, C; AANDRÉ TILLMAN, M. A.; VILLELA, F. A. Bioativador no desempenho fisiológico de sementes de arroz. Rev. bras. sementes, Londrina, v.33, n.3, p.501-510, 2011.

AVELAR, S. A. G.; BAUDET, L.; PESKE, S. T.; LUDWIG, M. P.; RIGO, G. A.; CRIZEL, R. L.; OLIVEIRA, S. Storage of soybean seed treated with fungicide, insecticide and micronutrient and coated with liquid and powered polymer. Ciência Rural, Santa Maria, 2011; v.41, n.10, p. 1719-1725, 2011.

BALARDIN, R. S.; SILVA, F. D. L.; DEBONA, D.; CORTE, G. D.; FAVERA, D. D.; TORMEN, N.R. Tratamento de sementes com fungicidas e inseticidas como redutores dos efeitos do estresse hídrico em plantas de soja. Ciência Rural, v.41, n.7, p.1120-1126, 2011.

CASTRO, G. S.; BOGIANI, J. C.; SILVA, M. G. GAZOLA, E.; ROSOLEM, C. A. Tratamento de sementes de soja com inseticidas e um bioestimulante. Pesquisa Agropecuária Brasileira, Brasilia, v.43, n.10, p.1311-1318, 2008.

CUNHA, R. P.; CORREA, M. F.; SCHUCH, L. O. B.; OLIVEIRA, R. C.; JUNIOR, J. S. A.; SILVA, J. D. G.; ALMEIDA, T. L. Diferentes tratamentos de sementes sobre o desenvolvimento de plantas de soja. Ciência Rural, Santa Maria, v.45, n.10, 2015.

DAN, L. G. M.; DAN, H. A.; BARROSO, A. L. L.; BRACCINI, A. L. Qualidade fisiológica de sementes de soja tratadas com inseticida sob efeito do armazenamento. Revista Brasileira de Sementes, Londrina, v. 32, n.2, p. 131-139, 2010.

KRZYZANOWSKI, F. C.; FRANÇA NETO, J. B.; COSTA, N. P. Teste do hipoclorito de sódio para semente de soja. Londrina: Embrapa Soja,( Circular Técnica, 37), 2004, 4p.

LUDWIG, M. P.; LUCCA FILHO, O. A.; BAUDET, L.; DUTRA, L. M. C.; AVELR, S. A. G.; CRIZEL, R. L. Qualidade de sementes de soja armazenadas após recobrimento com aminoácido, polímero, fungicida e inseticida. Revista Brasileira de Sementes, Londrina, v.33,n.3, p.395-406, 2011.

MAGUIRE, J. D. Speed of germination-aid in selection and evaluation for seedlig emergence and vigor. Crop Science, Madison, v.2, n.1, p.176-177,1962.

MERTZ, L. M.; ZIMMER, P. D.; HENNING, F. A. Bioprotetores e fungicidas químicos no tratamento de sementes de soja. Ciência Rural, Santa Maria, v.39, n.1, p.1318, 2009.

MONDO, V. H. V.; GOMES JUNIOR, F. G.;, PUPIM, T. L.; CICERO, S. M. Avaliação de danos mecânicos em sementes de feijão por meio da análise de imagens. Revista Brasileira de Sementes, v.31, n.2, p.27-35, 2009. 
NAKAGAWA, J. Testes de vigor baseados no desempenho das plântulas. In: Krzyzanowski, F.C.; Vieira, R.D.; França Neto, J.B. (ed.). Vigor de sementes: conceitos e testes. Londrina: ABRATES, 1999, cap. 2, p.1-24p.

PEREIRA, C. E., OLIVEIRA, J. A., GUIMARÃES, R. M., VIEIRA, A. R., EVANGELISTA, J. R. E.; OLIVEIRA, G. E. Tratamento fungicida e peliculização de sementes de soja submetidas ao armazenamento. Ciência e Agrotecnologia, v.35, n.1, p.158-164, 2011.

SEGALIN, S. R.; BARBIERI, A. P. P; HUTH, C.; BECHE, M. MATTIONI, N. M.; MERTZ, L. M. Physiological quality of soybean seeds treated with different spray volumes. Journal of Seed Science, v.35, n.4, p. 501-509, 2013.

SILVA, T. A.; SILVA, P. B.; SILVA, E. A. A.; NAKAGAWA, J.; CAVARIANI, C. Condicionamento fisiológico de sementes de soja, componentes de produção e produtividade. Ciência Rural, v.46, n.2, p.227-232, 2016.

SOUZA, D. C.;ALBUQUERQUE, M. C. F.; ZORATO, M. F.; CARVALHO, D. C. Análise de danos mecânicos e qualidade de sementes de algodoeiro. Revista Brasileira de Sementes, v.31, n.3, p.123-131, 2009.

VANZOLINI, S.; ARAKII, C. A. S.; SILVA, A. C. T. M.; NAKAGAWA, J. Teste de comprimento de plântula na avaliação da qualidade fisiológica de sementes de soja. Revista Brasileira de Sementes, Londrina, v.29, n.2, p. 90-96, 2007. 